\title{
Remitting Seronegative Symmetrical Synovitis with Pitting Edema ( $\left.\mathrm{RS}_{3} \mathrm{PE}\right)$ Syndrome Accompanied by Parkinson's Disease
}

\author{
Eiichi Ito ${ }^{1}$, Hiroshi Okamoto ${ }^{2}$, Atsuko Mochizuki ${ }^{1}$, Kuniko Ohara ${ }^{1}$, Maiko Kato ${ }^{1}$, \\ Yutaka Terashima ${ }^{3}$, Eiichi Tanaka ${ }^{2}$, Kae Takagi $^{2}$, Shinichiro Uchiyama ${ }^{1}$ and Makoto Iwata ${ }^{1}$
}

\begin{abstract}
We encountered two cases of $\mathrm{RS}_{3} \mathrm{PE}$ (remitting seronegative symmetrical synovitis with pitting edema) syndrome accompanied by Parkinson's disease (PD). Although the etiology of $\mathrm{RS}_{3} \mathrm{PE}$ syndrome is still unknown, several possible associations, such as malignancies and viral infections, have been reported; $\mathrm{RS}_{3} \mathrm{PE}$ syndrome is thought to be an autoimmune-mediated disorder. The present patients did not have any factors which are reported to be associated with $\mathrm{RS}_{3} \mathrm{PE}$. Whether or not the complication of $\mathrm{PD}$ and $\mathrm{RS}_{3} \mathrm{PE}$ syndrome is incidental needs to be further examined, and we discuss here the possible cause of association between $\mathrm{PD}$ and $\mathrm{RS}_{3} \mathrm{PE}$ syndrome, including dopamine agonists one of the anti-PD medications.
\end{abstract}

Key words: $\mathrm{RS}_{3} \mathrm{PE}$ syndrome, Parkinson's disease, edema, steroid, dopamine agonist

(DOI: 10.2169/internalmedicine.46.0029)

\section{Introduction}

Remitting seronegative symmetrical synovitis with pitting edema $\left(\mathrm{RS}_{3} \mathrm{PE}\right)$ syndrome was originally reported by McCarty et al in 1985, and it is characterized by relatively acute, elderly onset symmetrical arthritis with prominent pitting edema of the hand and foot, a fast response with steroid therapy, and negative rheumatoid factor (1).

Parkinson's disease (PD) is considered to be a neurodegenerative movement disorder with the following signs and symptoms during the course of disease: resting tremor, akinesia, rigidity at limb and/or trunk, instability of posture, among others. The etiology of PD is not fully understood. An autoimmune-mediated process in PD has rarely been reported and the role of autoimmunity in the pathogenesis of $\mathrm{PD}$ is still controversial $(2,3)$.

Edema is often observed during the course of PD. If other factors that could cause edema, such as congestive heart failure and renal dysfunction are excluded, edema in patients with PD is considered to originate from autonomic dysfunction that is one of the symptoms of PD or from drug- induced side effects of dopamine agonists.

We encountered two cases of PD with prominent edema of the hand and foot. At first, their edema was thought to be a pathophysiological process of PD. However, clinical evidence indicated that their edema was associated with $\mathrm{RS}_{3} \mathrm{PE}$ syndrome and dramatic improvement with steroid therapy was observed. Here, we present the course of two cases of $\mathrm{PD}$ and $\mathrm{RS}_{3} \mathrm{PE}$ syndrome and discuss the relationship between these two independent disorders.

\section{Case 1}

A 72-year-old Japanese man was admitted to our hospital in July 2000 with acute arthralgia and edema affecting the hands and legs accompanied by low-grade fever $\left(37.1^{\circ} \mathrm{C}\right)$ for the first ten days of the previous month. He had a history of urinary tract stone at the age of 55 and hypertension since the age of 70 . He had no significant family history including obvious neurological and rheumatic disorders. In 1993, he noticed resting tremor of his right hand, difficulty of writing, and claudicating gait of the right leg. He visited a local clinic, was diagnosed as possible PD, and treated with oral amantadine hydrochloride, trihexiphenidyl hydrochloride,

\footnotetext{
${ }^{1}$ Department of Neurology, Neurological Institute, Tokyo Women's Medical University, Tokyo, ${ }^{2}$ Institute of Rheumatology, Tokyo Women's Medical University, Tokyo and ${ }^{3}$ Department of Cardiology, Cardiological Institute, Tokyo Women's Medical University, Tokyo

Received for publication January 18, 2007; Accepted for publication April 12, 2007

Correspondence to Dr. Eiichi Ito, eiichi-neko@goo.jp
} 
levodopa/dopa-decarboxylase inhibitor and bromocriptine mesilate as anti-PD medication. Physical examination showed the tender articular swelling and redness in his bilateral shoulder, wrist, hand, knee and foot joint with marked pitting edema at the bilateral dorsum of hand, foot and pretibial area. Respiratory sound revealed velcro rale at bilateral lower lung fields. He had no symptoms or signs of collagen diseases including exanthema, muscle weakness, and Raynaud's phenomenon. Neurological findings revealed akinesia, intermittent resting tremor of the right arm and cog-wheel rigidity. Laboratory studies revealed an elevation of white blood cell count $(8,200 / \mu \mathrm{l})$, C-reactive protein $(8.97$ $\mathrm{mg} / \mathrm{dl}$; normal $<0.33)$ and erythrocyte sedimentation rate $(76$ $\mathrm{mm} / \mathrm{hr}$; normal $15<$, male). Serum total protein $(6.1 \mathrm{~g} / \mathrm{dl}$; normal <6.5) and albumin $(2.9 \mathrm{~g} / \mathrm{dl}$; normal <3.8) level were both slightly decreased, hepatic, renal function and electrolytes were normal. Thyroid function was normal. Serum brain natriuretic peptide (BNP) was normal $(15 \mathrm{pg} / \mathrm{ml}$; normal<20). Antinuclear antibody titer was 80 times higher than normal control, rheumatoid factor (RF) was negative. Matrix metalloproteinase-3 (MMP-3) was normal. Autoantibodies were negative, including anti-Sm antibody, anti-Scl70 antibody, anti-Jo-1 antibody, anti RNP antibody and anti SS-A/SS-B antibodies. HLA typing was A24/31 B52/61 Cw 10 DR4/16 DQ6. ECG and echocardiogram was normal and chest X-ray suggested interstitial pneumonitis at bilateral lower lung fields. No osteoclastic findings were detected on articular X-ray study. On gallium-scintigram, RI accumulation was found at bilateral lower lung fields, bilateral shoulder joint, left wrist joint and bilateral knee joint. Malignancy was not detected by general imaging study including CT or RI scintigraphy. At first, we diagnosed him as rheumatoid arthritis, and he was treated with salazosulfapyridine 500 $\mathrm{mg}$ /day for a week, and 1,000 mg/day for another one week followed by the addition of oral prednisolone (PSL) $5 \mathrm{mg} /$ day. After PSL was added, articular symptoms slightly improved. However, agranulocytosis developed 1 month after the administration of salazosulfapyridine, and treatment with salazosulfapyridine was terminated and the dose of oral PSL was increased to $15 \mathrm{mg} /$ day. Two days after the increase of oral PSL dose, his articular symptom, edema dramatically improved, and one week later, he was discharged from our hospital. We diagnosed the patient as having $\mathrm{RS}_{3} \mathrm{PE}$ syndrome rather than rheumatoid arthritis based on the following reasons: 1) negative RF, 2) symmetrical arthritic symptom with prominent pitting edema and 3) clinical course of dramatic recovery with PSL therapy. After discharge, PSL dose was gradually tapered to $5 \mathrm{mg} /$ day without relapse as of October 2006.

\section{Case 2}

A 59-year-old Japanese woman was admitted to our hospital in August 2006 for acute fever $\left(37.2^{\circ} \mathrm{C}\right)$, arthralgia and edema of the four limbs for 4 weeks. She had a history of type 2 diabetes mellitus (DM) since the age of 54 and was treated with glibenclamide and metformin hydrochloride. In

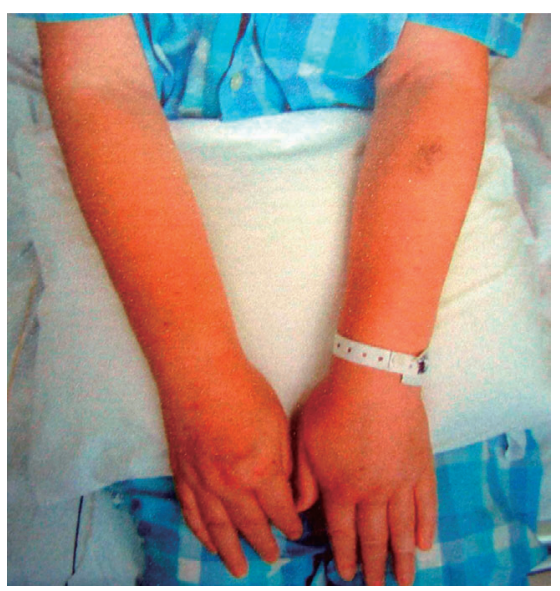

Figure 1. Photograph of the hands and forearm of Case 2 taken before starting oral prednisone administration. Pitting edema of the dorsum of hands, digits and forearm can be noted.

January 2004, akinesia, difficulty of writing, gait disturbance, cog-wheel rigidity in her right limb, and resting tremor of the right leg were noted. From these symptoms and signs, she was diagnosed as PD for the first time. She was treated with pramipexole hydrochloride hydrate in January 2005. Her parkinsonism improved partially after receiving therapy.

In July 2006, she noticed right shoulder pain and edema in the dorsum of her right hand. The patient assumed her articular symptoms were side effects of the medication. and she stopped taking her medication 2 weeks prior admission. At the time admission, physical examination revealed bilateral shoulder and upper arm pain, and heat with prominent pitting edema at the dorsum of her bilateral hand, feet and pretibial areas (Fig. 1). She had no symptoms or signs of collagen diseases including exanthema, muscle weakness, and Raynaud's phenomenon. Neurological findings revealed she had slight cog-wheel rigidity of her right upper and lower limb, resting tremor of her right leg, akinesia and truncal anteflexion posture. Laboratory studies revealed elevation of white blood cell count (WBC 9,370/ $\mu \mathrm{l}$ ), C-reactive protein was $12.1 \mathrm{mg} / \mathrm{dl}$ (normal <0.33) and erythrocyte sedimentation rate was $99 \mathrm{~mm} / \mathrm{hr}$ (normal <19, female). Serum total protein, serum albumin, liver function, renal function and electrolytes were normal. Thyroid function was normal. Fasting blood glucose $\left(263 \mathrm{mg} / \mathrm{dl}\right.$; normal <109) and $\mathrm{HbA}_{1 \mathrm{C}}$ $(10.2 \%$; normal <5.9) suggested poor control of her diabetes. Serum BNP was normal $(10 \mathrm{pg} / \mathrm{ml}$; normal $<20)$. Antinuclear antibody titer was 320 times higher than normal control, and rheumatoid factor (RF) was negative. MMP-3 was negative. Autoantibodies were negative, including antiSm antibody, anti-Scl-70 antibody, anti RNP antibody, antiJo-1 antibody and anti SS-A/SS-B antibodies. ECG and chest X-ray were normal. Echocardiogram revealed normal cardiac function. Bony destruction was not found in articular X-ray study. General Ga-scintigraphy found RI accumulation at the portion of peripheral joint of four extremities. Malignancy was not detected by CT scanning. She was diagnosed 
as $\mathrm{RS}_{3} \mathrm{PE}$ syndrome by her clinical profile and treated with oral PSL $20 \mathrm{mg} /$ day. Two days after PSL administration, her articular pain and edema improved, and after a week she had no symptoms. As of October 2006, PSL has been tapered, and her articular signs and symptoms have not relapsed.

\section{Discussion}

$\mathrm{RS}_{3} \mathrm{PE}$ syndrome is characterized by acute onset symmetrical synovitis and arthritis of limb, prominent edema especially in the dorsum of hand and foot, negative rheumatoid factor, elderly onset, good and fast response to corticosteroids and good prognosis that is a mono-phasic course and does not recur. Although the etiology of $\mathrm{RS}_{3} \mathrm{PE}$ syndrome is still unknown, possible association with $\operatorname{HLA}(1,4)$, malignancy (5-11), parvovirus B19 infection (12) have been proposed, and cases accompanied by other rheumatoid and autoimmune disorders have been reported (13-15). Therefore, it is estimated that autoimmunity plays a role in the pathogenesis of $\mathrm{RS}_{3} \mathrm{PE}$ syndrome. We were unable to detect any of the association factors of $\mathrm{RS}_{3} \mathrm{PE}$ to $\mathrm{PD}$. To our knowledge, cases of PD and $\mathrm{RS}_{3} \mathrm{PE}$ syndrome have not been reported to date. During the course of $\mathrm{PD}$, edema is sometimes experienced as a symptom of PD. If other edemacausing conditions, such as congestive heart failure, renal failure, general metabolic and endocrinological dysfunction are excluded, it is often suggested that edema in patient with $\mathrm{PD}$ is derived from autonomic dysfunction, and/or a sideeffect from drug therapy of PD, especially dopamine agonists. At first, we estimated that edema of our cases was derived by the factor associated with $\mathrm{PD}$ and/or medication. One report has shown that subjective edema appeared only on hands and feet, and the diagnosis of $\mathrm{RS}_{3} \mathrm{PE}$ syndrome was obtained by the quick improvement of edema using corticosteroid therapy (20). The report suggests that, even if articular symptom is not detected, $\mathrm{RS}_{3} \mathrm{PE}$ syndrome should not be excluded in the differential diagnosis of notable limb edema. In the case of edema with $\mathrm{PD}, \mathrm{RS}_{3} \mathrm{PE}$ syndrome may be one of the differential diagnoses and physical examination for arthritis, and the titer of general inflammation markers such as, CRP, WBC and ESR should be added.

We propose two possibilities regarding the association between PD and $\mathrm{RS}_{3} \mathrm{PE}$ syndrome: (1) direct association between $\mathrm{PD}$ and $\mathrm{RS}_{3} \mathrm{PE}$ syndrome, and (2) anti-PD druginduced $\mathrm{RS}_{3} \mathrm{PE}$ syndrome. There is no evidence showing the pathophysiological association between $\mathrm{PD}$ and $\mathrm{RS}_{3} \mathrm{PE}$ syndrome, thus far. In the present cases, both patients had taken dopamine D2 agonist prior to the diagnosis of $\mathrm{RS}_{3} \mathrm{PE}$ syndrome. Recent reports have discussed the relationship between increased serum vascular endothelial growth factor (VEGF) and the onset of $\mathrm{RS}_{3} \mathrm{PE}$ syndrome $(16,17)$. On the other hand, several reports suggest that dopamine D2 agonists inhibit the effect of VEGF by altering the phosphorylation state of its receptor, VEGFR-2 $(18,19)$. In the course of case 2, the patient's edema was present after stopping pramipexole administration, and the serum level of VEGF before the treatment with PSL was $1,010 \mathrm{pg} / \mathrm{ml}$, which is higher than those with other autoimmune disorders reported by Arima et al (16). Although we were unable to measure the serum concentration of VEGF in case 1, we speculated that discontinuous administration of dopamine D2 agonists might have played a role in the elevation of VEGF function and the development of $\mathrm{RS}_{3} \mathrm{PE}$ syndrome in case 2 . In addition, we speculate the following reason why no cases of coexistence of $\mathrm{RS}_{3} \mathrm{PE}$ syndrome with $\mathrm{PD}$ have reported thus far: most of the PD patients take dopamine D2 agonists for their treatment and this drug inhibits the effects of VEGF on the development of $\mathrm{RS}_{3} \mathrm{PE}$ syndrome, resulting in the low incidence of coexistence of $\mathrm{RS}_{3} \mathrm{PE}$ syndrome with $\mathrm{PD}$. On the other hand, case 1 had been taking D2 agonist before suffering from $\mathrm{RS}_{3} \mathrm{PE}$ syndrome. Therefore, the pathogenesis of $\mathrm{RS}_{3} \mathrm{PE}$ syndrome with PD is not fully understood yet and we speculated that unknown factors including the difference of each D2 agonist are involved in the pathogenesis. In conclusion, we encountered two cases of $\mathrm{RS}_{3} \mathrm{PE}$ syndrome with $\mathrm{PD}$, and VEGF may be the induction factor of $\mathrm{RS}_{3} \mathrm{PE}$ syndrome. Further accumulation of similar case reports might clarify the association between $\mathrm{PD}$ and $\mathrm{RS}_{3} \mathrm{PE}$ syndrome and the common factors involved in the pathogenesis of the two different disorders.

\section{References}

1. McCarty DJ, O’Duffy JD, Pearson L, et al. Remitting seronegative symmetrical synovitis with pitting edema. RS3PE syndrome. JAMA 15: 2763-2767, 1985.

2. Kuhn W, Muller T, Nastos I, Poehlau D. The neuroimmune hypothesis in Parkinson's disease. Rev Neurosci 8: 29-34, 1997.

3. Moller A, Perrild H, Pedersen H, Hoier-Madsen M. Parkinson's disease and autoimmunity. Acta Neurol Scand 79: 173-175, 1989.

4. Pease C, Bhakta B. RS3PE syndrome. The relation with HLA B7. Ann Rheum Di 59: 239-240, 2000.

5. Sibilia J, Friess S, Schaeverbeke T, et al. Remitting seronegative symmetrical synovitis with pitting edema (RS3PE): a form of paraneoplastic polyarthritis? J Rheumatol 26: 115-120, 1999.

6. Ekenel M, Yavuz S, Karti S, et al. Remitting seronegative symmetrical synovitis with pitting oedema associated with chronic lymphocytic leukaemia. Clin Rheumatol 19: 247-248, 2000.

7. Vinci M, Malaguarnera L, Pistone G. RS3PE and ovarian cancer. Ann Rheum Dis 60: 429-431, 2001.

8. Manganelli P, Delsante G, Bianchi G, et al. Remitting seronegative symmetrical synovitis with pitting oedema in a patient with myelodysplastic syndrome and relapsing polychondritis. Clin Rheumatol 20: 132-135, 2001.

9. Mouly S, Berenbaum F, Kaplan G. Remitting seronegative symmetrical synovitis with pitting edema following intravesical bacillus Calmette-Guerin instillation. J Rheumatol 28: 1699-1701, 2001.

10. Yamasaki T, Takeoka Y, Nakao Y, Fusamoto H. Remitting seronegative symmetrical synovitis with pitting edema (RS3PE) syndrome with malignant lymphoma. Nippon Naika Gakkai Zasshi 
91: 1031-1033, 2002 (in Japanese).

11. Tunc SE, Arslan C, Ayvacioglu NB, et al. Palaneoplastic remitting seronegative symmetrical synovitis with pitting edema (RS3PE syndrome): a report of two cases and review of the literature. Rheumatol Int 24: 234-237, 2004.

12. Perandones CE, Colmegna I, Arana RM. Parvovirus B19: another agent associated with remitting seronegative symmetrical synovitis with pitting edema. J Rheumatol 32: 389-390, 2005.

13. Cimmino MA, Silvestri E, Garlaschi G. Remitting seronegative symmetrical synovitis with pitting oedema (RS3PE) as recurrence of aborted PMR. Ann Rheum Dis 60: 303, 2001.

14. Choi YM, Sheen DH, Lee YJ, et al. Sjogren's syndrome presenting as remitting seronegative symmetrical synovitis with pitting edema (RS3PE). J Korean Med Sci 18: 606-608, 2003.

15. Kato T, Ubara Y, Sawa N, et al. An abrupt onset of seropositive polyarthritis. with prominent distal tenosynovitis concomitant with bronochiolitis obliterans organizing pneumonia (BOOP): consideration of the relationship with RS3PE syndrome. Intern Med 43: 143-147, 2004.
16. Arima K, Origuchi T, Tamai M, et al. RS3PE syndrome presenting as vascular endothelial growth factor associated disorder. Ann Rheum Dis 64: 1653-1655, 2005.

17. Matsuda M, Sakurai K, Fushimi T, et al. Sarcoidosis with high serum levels of vascular endothelial growth factor (VEGF), showing RS3PE-like symptoms in extremities. Clin Rheumatol 23: 246248, 2004.

18. Mayuzumi T. Vascular endothelial growth factor (VEGF) production by a dopamine D2 receptor agonist and its action mechanism in glioma cells. Hokkaido Igaku Zasshi 80: 115-123, 2005.

19. Gomez R, Gonzalez-Izquierdo M, Zimmermann R, et al. Lowdose dopamine agonist administration blocks vascular endothelial growth factor (VEGF)-mediated vascular hyperpermeability without altering VEGF receptor 2-dependent luteal angiogenesis in a rat ovarian hyperstimulation model. Endocrinology 147: 54005411, 2006.

20. Coiteaux I, Depairon M, Hayoz D. Pitting edema of the four extremities of rapid appearance. Vasa 34: 144-146, 2005.

(C) 2007 The Japanese Society of Internal Medicine http://www.naika.or.jp/imindex.html 